\title{
Эмпирическое тестирование теорий структуры капитала: модели, направления, результаты
}

\author{
Зинкевич Н.B. ${ }^{69}$, Олеванова Е.A. ${ }^{70}$
}

Данная статья представляет собой обзор исследований, посвященных эмпирическому тестированию выбора компаниями структуры капитала. Рассматриваются работы, проверяюшие теории компромисса (trade-off theory) и порядка финансирования (pecking order theоry) на развитых и развиваюшихся рынках. Большое внимание уделяется эконометрическим проблемам данного класса исследований и критическому анализу их результатов. В числе методов тестирования теорий структуры капитала рассматриваются новые подходы: динамическая теория компромисса с горизонтом бездействия и метод событий (event study).

\section{JEL: $G 32$}

Ключевые слова: обзор исследований, корпоративные финансы, структура капитала, теория компромисса, теория порядка финансирования

\section{Введение}

Проблема выбора оптимального соотношения собственного и заемного капитала актуальна не только для каждой компании, менеджеры которой на определенном этапе ее развития должны делать сознательный выбор между источниками финансирования, но и для финансовой системы в целом. Слабое развитие финансовых рынков и невозможность привлечения определенного типа финансирования способны подавлять инвестиционную активность фирм и замедлять развитие всей экономики.

Основы теории финансирования фирмы были заложены Модильяни и Миллером [Modigliani, Miller, 1958], которые в своей теоретической работе показали, что выбор между долгом и собственным капиталом не оказывает влияния на стоимость привлечения капитала и стоимость фирмы. Особенность их теории было большое число ограничительных предпосылок: отсутствие налогов и информационной асимметрии, транзакционных издержек, однородность инвесторов, совершенный финансовый рынок.

Авторы ранних работ искали объяснение выбора фирмы между заемным и внутренним финансированием в конъюнктуре финансовых рынков. Так, Бэкстер и другие [Baxter et.al., 1970] исследовали зависимость между решением фирмы выпустить облигации или акции и ожидаемой доходностью ценных бумаг, а также текущей структурой капитала фирмы.

Зависимость между текущим уровнем долга фирмы и ее способностью привлекать заемный капитал в дальнейшем легла в основу первой из основных теорий финансирования, так называемой компромиссной теории (trade-off theory). Согласно ей, фирмы выбирают оптимальное соотношение собственного и заемного капитала, при которой предельные выгоды налогового щита, образованного выплатами по долгу, равняются издержкам финансовой неустойчивости (costs of financial distress), которые тем больше, чем выше текущий уровень долга. Измерению издержек финансовой неустойчивости и их значимости посвящен целый ряд работ [Weiss, 1990; Andrade, Kaplan, 1998; Gilson, 1997; Almeida, Philippon, 2006]. Как отмечают Грэхэм и Харви [Graham, Harvey, 2001], соображения

\footnotetext{
${ }^{69}$ Магистр 1-го курса программы «Стратегическое управление финансами фирмы», ГУ ВШЭ, стажер отдела оценки бизнеса ООО «Эрнст энд Янг - оценка».

${ }^{70}$ Магистр 1-го курса программы «Стратегическое управление финансами фирмы», ГУ ВШЭ.
}

Выпуск \#1(5), 2008

(C) Электронный журнал Корпоративные Финансы, 2008 
налоговой оптимизации играют в США важную роль в принятии решений финансовыми менеджерами.

Теория порядка финансирования (pecking order theory) снимает другое введенное Модильяни и Миллером ограничение: о симметрии информации между инвесторами и менеджерами корпорации. Как отмечает Дональдсон [Donaldson, 1961], компании прежде всего используют в качестве источника финансирования нераспределенную прибыль и лишь в случае необходимости переходят к ценным бумагам с наименьшим риском - облигациям, на цены которых информационная асимметрия оказывает менее негативное влияние. Теория порядка финансирования получила развитие в работах Майерса и Майлуфа [Myers, Majluf, 1984] и Pocca [Ross, 1977].

Целью данной работы является обзор основных исследований, посвященных эмпирической проверке релевантности компромиссной теории и теории порядка финансирования для объяснения финансовых решений, принимаемых фирмами на практике. В первом разделе формулируются основные статистические закономерности, подлежащие проверке в рамках обеих теорий. Во втором разделе рассматриваются основные исследований, посвященные тестированию теорий структуры капитала в статическом аспекте. В третьем разделе описываются основные результаты проверки теорий в динамическом аспекте. Четвертый раздел посвящен критическому анализу моделей, традиционно используемых для тестирования теорий структуры капитала, а также описанию двух новых направлений исследований: динамических моделей компромисса с горизонтом бездействия и методу изучения событий (event study). В заключении, помимо выводов о текущем состоянии дел в области тестирования теорий структуры капитала, обсуждается возможность проведения подобных исследований на российском финансовом рынке.

Большое внимание в обзоре уделяется статистическим аспектам эмпирических исследований: выбору прокси-переменных (proxy) при невозможности в явном виде измерить тот или иной фактор, подбору оптимальной модели, мощности используемых тестов.

\section{1. Факторы, определяющие выбор фирм в рамках теорий компромисса и порядка финансирования}

Компромиссная модель утверждает, что фирма, которая максимизирует свою стоимость, будет выбирать оптимальную структуры капитала путем взвешивания издержек и выгод от дополнительной единицы финансирования и в итоге выберет ту долю заемного капитала, при которой предельные издержки равны предельным выгодам. Выгоды от заемного капитала включают в себя налоговые преимущества и меньшие агентские издержки от свободного денежного потока, издержки использования заемного капитала включают увеличивающийся риск финансовой неустойчивости (оплата юридических и консультационных услуг при проведении процедуры банкротства, потеря поставщиков, покупателей, иных контрагентов).

Как следствие, наблюдаемый уровень финансового рычага положительно зависит от величины налогового щита и отрицательно - от величины издержек финансовой неустойчивости. Эту зависимость можно исследовать как на пространственных выборках (много фирм в один и тот же период времени), так и по данным временных рядов (выбор одной и той же фирмы в разные периоды). Авторы исследований, которым посвящен данных обзор, используют панельные данные, что позволяет увеличить число наблюдений.

Величина налогового щита может измеряться эффективной ставкой налога на прибыль. Как подчеркивает Тауб [Taub, 1975], установленная законодательством налоговая ставка в данном случае неприменима, поскольку налоговые льготы, штрафы или накопленные убытки могут привести к тому, что официальная ставка налога не совпадает с той, по которой компания осуществляет платежи в реальности. Бут и другие [Booth et.al., 2001] отмечают, что эффективная ставка налога на прибыль может быть плохой прокси- 
переменной для налоговых выгод ввиду использования схем налоговой оптимизации. Поэтому иные авторы [Chen, 2004; Loof, 2004] используют для измерения налоговых выгод величину недолговых налоговых щитов (амортизацию), которые, согласно теории, должны снижать оптимальный уровень долга компании. Брэдли и другие [Bradley, 1984; et.al.] замечают, что и эта прокси-переменная неидеальна: большую величину амортизации имеют, как правило, фирмы с высокой долей материальных активов, уровень оптимального долга для которых выше.

Лонг и Мэлитц [Long, Malitz, 1985] отмечают, что для фирм с высокой долей материальных активов издержки финансовой неустойчивости ниже, поскольку для таких компаний репутация имеет меньшее значение. Оплер и Титман [Opler, Titman, 1994] показывают, что фирмы с высокой долей нематериальных активов и инвестиций в разработки несут высокие издержки финансовой неустойчивости и часто теряют рыночные позиции при резком увеличении финансового рычага. Кроме того, под залог материальных активов фирма может привлечь более дешевый кредит, что усиливает положительную зависимость между долей материальных активов в общих активах и привлекательностью заемного финансирования.

Фрэнк и Гойэл [Frank, Goyal, 2002], Лонг и Мэлитц показывают, что фирмы с большими возможностями роста (темп роста продаж за последние несколько лет) выбирают низкие уровни долга, поскольку стоимость долга для них слишком высока. Альтернативными мерами возможностей роста являются Q Тобина (отношение рыночной стоимости фирмы к балансовой) [Norvaišienè \& Stankevičienė, 2007] или коэффициент P/E (отношение цены к прибыли на акцию) [Adedeji, 2001]. Как отмечают Бут и другие, оценки возможностей роста, построенные на рыночных котировках компании, сильно зависят от краткосрочных колебаний фондового рынка, поэтому их использование в регрессиях нежелательно. Между финансовым рычагом, в знаменателе которого стоит рыночная стоимость собственного капитала, и Q Тобина по определению будет наблюдаться отрицательная зависимость, которая не имеет никакой объясняющей силы.

Раджан и Зингалес [Rajan, Zingales, 1995] и ряд других авторов справедливо предполагают, что между оптимальным уровнем долга и размером фирмы существует положительная зависимость, поскольку для крупных фирм заемное финансирование более доступно. Доступность заемного финансирования также положительно связана с прибыльностью компании, измеряемой как доходность на активы (EBITDA/Активы).

Кроме того, авторы ряда работ пытаются ввести прокси-переменную величины издержек финансовой неустойчивости в регрессию напрямую [Delcoure, 2007; Booth et. al. 2001; Taub, 1975]. Как правило, для этих целей используется волатильность операционной прибыли компании, рассчитанная как стандартное отклонение показателя ЕВІТ или как среднее абсолютное отклонение.

Теория порядка финансирования основывается на гипотезе о том, что асимметричная информация создает иерархию издержек внешнего финансирования. Новые инвестиционные проекты финансируются в первую очередь за счет собственных доходов (retentions), затем за счет низкорисковых долговых обязательств, конвертируемых долговых обязательств, собственного капитала в последнюю очередь. В каждый момент времени существует оптимальное решение по выбору способа финансирования, которое зависит от чистого денежного потока, определяемого доступные фонды. В соответствии с этой теорией, фирмы гораздо чаще будут прибегать к заимствованиям, чем к использованию собственного капитала, если внутреннего денежного потока не хватает, чтобы покрыть расходы на капитал. Данная теория не определяет единственного оптимального решения по структуре капитала, к которому фирма стремилась бы в долгосрочном периоде.

Величина финансового рычага в теории порядка финансирования определяется в первую очередь величиной дефицита внутренних средств у компании. Шайм-Сандер и Майерс [Shyam-Sunder, Myers, 1999] предлагают измерять его как разницу операционного денежного потока и всех осуществляемых из него денежных выплат: 


$$
D E F_{i t}=D I V_{i t}+X_{i t}+\Delta W_{t}+R_{t}-C_{t},
$$

где

DIV - дивидендные платежи,

$\mathrm{X}$ - капитальные расходы,

$\Delta W$ - прирост оборотного капитала,

$\mathrm{R}$ - часть долгосрочного долга, которая должна быть выплачена в этом периоде,

$\mathrm{C}$ - операционный денежный поток (EBIT).

Дефицит финансовых средств вводится в регрессии в отношении к активам, чтобы устранить влияние масштаба. Шайм-Сандер и Майерс справедливо отмечают, что смысл имеют как положительные, так и отрицательные значения дефицита: при избытке свободных средств фирма может выкупить часть выпущенного долга и вернуться к финансированию за счет внутренних источников. Адедеджи [Adedeji, 2001], наоборот, утверждает, что выкуп долга для фирм нехарактерен, поэтому отрицательные значения дефицита не имеют объясняющей силы, и обнуляет их, что делает выборку цензурированной.

Теория порядка финансирования утверждает, что финансовый дефицит должен полностью покрываться выпуском дополнительного долга, но на практике коэффициент при этом регрессоре далек от единицы [Loof, 2004; Nivorozhkin, 2004]. По мнению ШаймСандера и Майерса, этот результат не следует рассматривать как провал теории порядка финансирования, поскольку оптимальному реагированию фирм препятствуют транзакционные издержки. Эти же исследователи отмечают, что идеальным вариантом было бы использовать в проверке не текущее значение дефицита, а ожидаемое в будущем периоде, на которое фирма на самом деле ориентируется, принимая финансовые решения.

Размер фирмы в теории порядка финансирования оказывает на финансовый рычаг отрицательное влияние, поскольку крупные фирмы сталкиваются на рынках акций с меньшей информационной асимметрией и потому быстрее переходят от долга к выпуску акций [Titman, Wessels, 1988]. Более прибыльные фирмы дольше не сталкиваются с финансовым дефицитом и финансируют инвестиции за счет нераспределенной прибыли, поэтому уровень их долга ниже [Kester, 1986; Shyam-Sunder, Myers, 1999].

Возможности роста оказывают на решения в рамках теории порядка финансирования двоякий эффект: с одной стороны, фирмы с высокими возможностями роста более прибыльны и сталкиваются с высокими издержками выпуска долга, что приводит к выбору низкого уровня финансового рычага [Chen, 2004]. С другой - быстрорастущие фирмы часто испытывают недостаток средств для инвестиций, что стимулирует их выход на долговой рынок.

Зависимой переменной при тестировании моделей структуры капитала является финансовый рычаг (отношения долга к стоимости фирмы или собственному капиталу) или, в отдельных случаях, переменная бинарного выбора фирмы между способами финансирования (выпуск долга или акций). В отношении способа измерения финансового рычага среди исследователей нет единства: хотя рыночный (market) финансовый рычаг, в котором собственный капитал учитывается по капитализации, является впередсмотрящим (forwardlooking) среди переменных и лучше подходит в качестве ориентира для принятия финансовых решений [Booth et.al., 2001], по ряду рынков данные о капитализации недоступны или не могут использоваться в исследованиях.

Так, Матеус и Терра [Mateus, Terra, 2005] работают с данными по компаниям Латинской Америки и Восточной Европы, рынки акций которых неликвидны, и потому используют бухгалтерский финансовый рычаг (book leverage), в который собственный капитал входит по балансовой стоимости. Таким же образом поступает Чен [Chen, 2004]. Как отмечают Раджан и Зингалес [Rajan\&Zingales, 1995], при проведении исследования по компаниям разных стран необходимы корректировки на различия в системах бухгалтерского учета (системы начисления резервов, признания пенсионных обязательств), в противном случае финансовые рычаги будут несопоставимы. $\mathrm{K}$ сожалению, большинство исследователей такие корректировки не проводят.

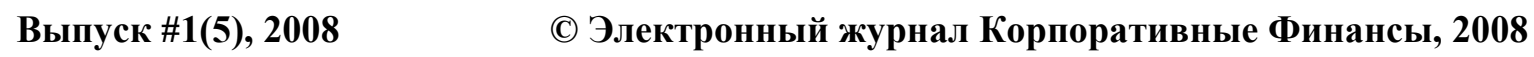


В зависимой от переменной используется как уровень общего финансового рычага (отношение совокупного долга к стоимости компании), так и уровень долгосрочного финансового рычага. Результаты регрессий иногда оказываются различными, причем различия особенно велики для развивающихся рынков [Mateus, Terra, 2005]. Как отмечает Чен [Chen, 2004], несоответствие результатов обусловлено тем, что для финансирования инвестиций компании развивающихся рынков вынуждены использовать краткосрочный долг, а уровень долгосрочного долга крайне низок и не может быть оптимальным, поскольку компании сталкиваются с ограничениями на привлечение «длинных денег».

Когда зависимой переменной является финансовый рычаг, тестирование осуществляется по панельным данным. Для выбора между сквозной регрессией, моделью с фиксированными эффектами (FE) и моделью со случайными эффектами (RE) используются стандартные методики: тест Хаусмана и тест на систему ограничений. Если зависимая переменная бинарная [Baxter et. al., 1970; Chun, Smith, 2002], регрессия строится по временным рядам.

\section{2. Тестирование статической теории компромисса против теории порядка финансирования}

Тестирование релевантности теорий trade-off и pecking order проводится в подавляющем большинстве исследований параллельно. Это связано с тем, что обе теории предполагают влияние сходного набора факторов на оптимальное значение финансового рычага, но направление влияния, как правило, отличается. В ряде ранних работ [Taub, 1975; Bradley et. al., 1984] тестируется только теория компромисса, поскольку теоретические основы теории порядка финансирования на тот момент были не проработаны.

В исследованиях используется информация по выборке компаний из одной [Bradley et. al., 1984; Chen, 2004; Zou, Xiao, 2006] или нескольких [Rajan, Zingales, 1995; Booth et.al. 2001; Delcoure, 2007] стран за некоторый период (годовые данные). Общий вид регрессионного уравнения следующий:

$$
L_{i t}=a_{i t}+X_{i t} * \beta+\varepsilon_{i t}
$$

где

$\mathrm{L}_{\mathrm{it}}$ - финансовый рычаг компании і периоде $\mathrm{t}$,

Xit - вектор факторов, определяющих значение L, для компании і в периоде t,

$\mathrm{b}$ - вектор коэффициентов при факторах (именно они представляют основной интерес для исследователя),

$\mathrm{a}_{\mathrm{it}}$ - свободный член регрессии.

Также нужно отметить, что во всех работах изучается только структура капитала компаний нефинансового сектора. Финансовые компании обладают специфической структурой активов и пассивов, долг для них является не способом привлечения финансирования, а «сырьем» [Дамодаран, 2005]. Структура капитала финансовых компаний определяется главным образом регулятивными требованиями к капиталу, но не теориями компромисса или порядка и финансирования, поэтому из общей выборки их необходимо исключить [Rajan, Zingales, 1995].

Раджан и Зингалес строят эмпирическое исследование на данных о приблизительно 8000 компаний из стран «большой семерки» за период 1987-1991 годов, взятых из базы Global Vantage. Особенность этой работы состоит в том, что авторы не используют панельных данных в явном виде, а усредняют информацию по компаниям за четыре года наблюдения и проводят тестирование по этим данным, получая в чистом виде пространственную выборку.

Авторы проводят набор корректировок к бухгалтерской отчетности компаний, позволяющих выделить именно те элементы собственного и заемного капитала, которые используются для финансирования инвестиций:

1. Из активов и пассивов вычитаются наличность и наиболее ликвидные обязательства. 
2. Для Германии пенсионные обязательства вычитаются из обеих сторон баланса, чтобы добиться соответствия с остальными странами, где пенсионные обязательства на балансе не отражаются.

3. Отложенные налоги переносятся в собственный капитал.

4. Из активов вычитается балансовая стоимость нематериальных активов (goodwill и другие); авторы объясняют это тем, что произошедшая в США в 1980-х годах волна слияний и поглощений могла привести к переоцененности компаний, и эта переоцененность нашла отражение в размере нематериальных активов на балансе. ${ }^{71}$

5. Резервы для обеспечения будущих обязательств германских компаний, по словам авторов, есть форма акционерного капитала, поэтому они переносятся в собственный капитал.

По мнению Раджана и Зингалеса, проведение корректировок позволяет устранить недостатки предыдущих работ в той же области [Borio 1990; Frankel, Montgomery, 1991], авторы которых находили большие различия в уровнях финансового рычага по странам, которые не объяснялись ни одной из введенных в регрессию переменных.

Раджан и Зингалес выделяют следующие факторы, способные, согласно теориям структуры капитала, влиять на величину финансового рычага компаний: доля материальных активов (отношение постоянных активов к общим), Q Тобина, размер фирмы, прибыльность. В анализе отсутствует изучение таких важных аспектов теории компромисса, как размер налогового щита и издержки финансовой неустойчивости.

Для оценки регрессии авторами используется Тобин-модель и метод максимального правдоподобия, поскольку ввиду корректировок к финансовой отчетности выборку пришлось цензурировать, удалив отрицательные значения финансового рычага.

Раджан и Зингалес обнаруживают положительную зависимость между финансовым рычагом и долей материальных активов в общих активах, отрицательную зависимость между возможностями роста и прибыльностью компании и её финансовым рычагом (что согласуется с теорией порядка финансирования), положительную зависимость между размером фирмы и финансовым рычагом (согласуется с теорией компромисса) для всех стран, кроме Германии. Последний результат является труднообъяснимым, поскольку законодательство, регулирующее процедуру банкротства, в Германии намного строже, чем в других развитых странах. Как отмечают Уайт [White, 1993] и Кайзер [Kaiser, 1994], процедура банкротства в Германии ведет к ликвидации компании, а не к реорганизации, как, например, в США. Влияние институциональной системы на уровень финансового рычага не отмечается: статистическая разница между странами с ориентированной на рынок (marketoriented) и ориентированной на банки (bank-oriented) финансовыми ситемами отсутствует.

Работы Тауба [Taub, 1975] и Брэдли и других [Bradley et.al., 1984] не исследуют межстрановые различия в уровне финансового рычага, но обладают определенным преимуществом перед работой Раджана и Зингалеса: в них в явном виде вводятся в качестве объясняющих переменных величина налогового щита и прокси-переменная для издержек финансовой неустойчивости. Брэдли и другие получают полное подтверждение выводов теории компромисса на данных США, Тауб же отмечает необъяснимую в рамках данной теории положительную зависимость между размером недолговых налоговых щитов и уровнем долга.

Бут и другие [Booth et.al., 2001] проводят исследование по компаниям из 10 развивающихся стран за период 1980-1991 годов. Данные они получают из базы IFC (International Finance Corporation), содержащей информацию по 100 крупнейшим компаниям каждой страны. Таким образом, выборка изначально оказывается смещенной в сторону крупнейших компаний; кроме того, по странам эти компании могут быть разного размера. Авторы отдельно рассматривают общий и долгосрочный финансовый рычаги, поскольку

\footnotetext{
${ }^{71}$ Rajan\&Zingales [1995], p. 1434.
}

Выпуск \#1(5), 2008 
компании развивающихся рынков часто используют для финансирования инвестиций краткосрочный долг.

В регрессию, помимо переменных, использованных Раджаном и Зингалесом, включаются переменные, характеризующие уровень операционного риска бизнеса, и эффективная налоговая ставка. Бут и другие отмечают, что в качестве меры налогового преимущества долга корректнее было бы использовать коэффициент Миллера [Miller, 1997], учитывающий информацию о ставках налогов на доходы физических лиц, но не делают этого из-за отсутствия информации по ряду стран.

Результаты исследований Бута и других полностью опровергают теорию компромисса: уровень материальности активов незначим для общего финансового рычага, который отражает реальный уровень долга, используемый для финансирования инвестиций на развивающихся рынках, размер фирмы и уровень операционного риска на финансовый рычаг не влияют, а эффективная ставка налога оказывает негативное воздействие на прибыль. По мнению авторов, эффективная ставка налога является мерой прибыльности компании, поэтому знак зависимости может объясняться теорией порядка финансирования. Прямая мера прибыльности - доходность активов - также отрицательно связана с финансовым рычагом.

В выборке, использованной Бутом и другими, китайские компании отсутствуют, поэтому представляют большой интерес исследования Чена [Chen, 2004], Марсдена и Верарагавана [Marsden, Veeraraghavan, 2005] и Зоу, Ксиао [Zou, Xiao, 2006], проведенные исключительно по китайским данным. В работе Чена используются данные за шестилетний период (с 1995-го по 2000 год) по 77 публичным компаниям, торгующимся на Шанхайской фондовой бирже. Как отмечает сам автор, его выборка значительно смещена в сторону крупных промышленных компаний. Чен исследует влияние на финансовый рычаг тех же факторов, что и Бут и другие, но вместо средней ставки налога на прибыль использует недолговые налоговые щиты для оценки налоговых выгод долга.

Обращает на себя внимание следующий результат данной работы: если для общего финансового рычага выделенные факторы имеют большую объясняющую силу $\left(\mathrm{R}^{2}\right.$ достигает $0,6)$, то для долгосрочного рычага объясняющая сила крайне слабая $\left(\mathrm{R}^{2}\right.$ менее 0,1$)$. Вероятно, существуют не учтенные в анализе факторы, такие, как степень аффилированности с банками или доля собственности государства в компании, влияющие на уровень финансового рычага.

Чен получает результаты в пользу теории порядка финансирования, основные факторы теории компромисса (уровень операционного риска, налоговые щиты) оказываются незначимыми. Теория компромисса абсолютно нерелевантна, во-первых, потому, что компании получают целевое финансирование через государственные банки; во-вторых, эффекты налогового щита не имеют значения для государственных компаний, прибылью которых, как и налоговыми поступлениями, распоряжается государство.

Чен говорит о существовании «новой теории порядка финансирования» (сначала нераспределенная прибыль, потом акции и только потом долговой капитал из-за неразвитости долгового рынка) для китайских фирм. Ксиаокиу [Xiaoqui 2004] обращает внимание на уникальную особенность китайской экономики: наличие большого количества неторгуемых акций, владельцы которых стремятся максимизировать балансовую стоимость своих инвестиций путем выпуска новых акций. По мнению данного автора, этот институциональный механизм способствует формированию «нового порядка финансирования» китайских компаний.

Марсден и Верарагаван [Marsden, Veeraraghavan, 2005] проводят тестирование гипотезы Ксиакиу, вводя в регрессию в качестве объясняющей переменной долю владельцев неторгуемых акций. Авторы отмечают, что владельцы неторгуемых акций могут влиять на принятие решений в компании, только когда их доля велика, поэтому строится второй вариант регрессии с тремя фиктивными переменными (высокая, средняя, низкая доли). 
Результаты исследования подтверждают предположение Ксиаокиу, другие факторные зависимости укладываются в рамки теории порядка финансирования.

Недавнее исследование, проведенное Зоу и Ксиао [Zou, Xiao, 2006], показывает, что со временем для китайских фирм могла стать более актуальной теория компромисса: финансовый рычаг имеет положительную зависимость от размера фирм и доли материальных активов в общих. Тонг и Грин [Tong, Green, 2004] получают противоречивые результаты: хотя выбор уровня долга китайскими фирмами хорошо описывается теорией порядка финансирования, механизм принятия инвестиционных решений ей противоречит. Чем выше уровень долга компании, тем выше в среднем ее инвестиционная активность, в то время как теория порядка финансирования подразумевает обратную зависимость ввиду агентских проблем, в частности «нависания долга» (debt overhang) [Grinblatt, Titman, 2002].

\section{3. Тестирование динамической теории компромисса против теории порядка финансирования}

Динамическая теория компромисса предполагает, что у компании существует некий оптимальный уровень финансового рычага (определяется соотношением выгод налогового щита и издержек финансовой неустойчивости), к которому она по мере возможности подстраивается в каждом периоде. Невозможность мгновенной подстройки финансового рычага обусловлена неопределенностью, с которой сталкивается фирма, и транзакционными издержками приспособления (затраты на принятие решения о выпуске ценных бумаг, оплата услуг финансовых посредников и т.д.) Оптимальный уровень долга, как и скорость приспособления, меняется со временем.

В целом динамику финансового рычага в теории trade-off можно описать следующим уравнением [Shyam-Sunder, Myers, 1999]:

$$
L_{i t}-L_{i t-1}=a+b_{i t}\left(L^{*}{ }_{i t}-L_{i t-1}\right)+\varepsilon_{i t} \text {, }
$$

где

$\mathrm{L}_{\text {it }}-$ финансовый рычаг компании і в периоде $\mathrm{t}$,

$\mathrm{L}_{\text {it }}$ - оптимальный уровень рычага компании і в периоде $\mathrm{t}$,

$b_{\text {it }}$ - скорость приспособления к оптимальному уровню долга компании і в периоде $t$.

Теория компромисса предполагает, что коэффициент b должен лежать в интервале от 0 до 1. Чем он ниже, тем более высоки для компании издержки приспособления. Данная спецификация регрессии имеет несколько проблем: в качестве одного из факторов используется лаговая зависимая переменная, что ведет к необходимости использовать обобщенный метод моментов вместо метода наименьших квадратов [Mateus, Terra, 2005]; авторегрессионное поведение финансового рычага может объясняться не только теорией компромисса; способ определения оптимального финансового рычага неоднозначен. ШаймСандер и Майерс используют в качестве $L^{*}$ историческое среднее либо скользящую среднюю, Ниворожкин [Nivorozhkin, 2004] и Лооф [Loof, 2004] рассматривают дополнительные регрессионные модели для определения $L^{*}{ }_{i t}$ и $b_{i t}$ в каждом периоде и оценивают систему из трех регрессий, используя нелинейную процедуру оценки, Джилсон [Gilson, 1997] напрямую подставляют в регрессию линейную комбинацию факторов, поеределяющих L* в статических моделях. Наконец, Матеус и Tерра [Mateus, Terra, 2005] проводят оценку уравнения (3) в разностях.

Теория порядка финансирования предполагает, что изменения в уровне финансового рычага компании вызываются потребностью во внешнем финансировании (внутренним дефицитом):

$$
\mathrm{L}_{\mathrm{it}}-\mathrm{L}_{\mathrm{it}-1}=\mathrm{a}+\mathrm{b} * \mathrm{DEF} \mathrm{it}_{\mathrm{it}}+\mathrm{u}_{\mathrm{it}}
$$

где

$\mathrm{DEF}_{\text {it }}$ - внутренний дефицит компании в отношении к активам.

Рядом исследователей были получены результаты в пользу динамической теории компромисса. Так, Таггарт [Taggart, 1977], Марш [Marsh, 1982], Оплер и Титман [Opler,
Выпуск \#1(5), 2008
() Электронный журнал Корпоративные Финансы, 2008 
Titman, 1994] показывают, что финансовые рычаги фирм имеют тенденцию возвращения к среднему (mean reversion), то есть существует некий оптимальный уровень долговой нагрузки. Марш использует логит-модель, связывающую вероятность выпуска акций или облигаций с размером отклонения текущего значения долга от оптимального, которое измеряется как среднее за период наблюдения. Оплер и Титман также используют модель бинарного выбора, но оптимальный уровень долга оценивается на основе дополнительной регрессии. Ховакимиан, Оплер и Титман [Hovakimian, Opler, Titman, 2001] дополняют результаты более ранней работы Оплера и Титмана и анализируют не только решения о выпуске новых ценных бумаг, но и решения о выкупе акций или досрочном погашении облигаций. Джелилвэнд и Харрис [Jalilvand, Harris, 1984] и Ауэрбах [Auerbach, 1985] оценивают регрессию вида (3) и получают значимые и близкие к единице коэффициенты подстройки $b$.

Особого внимания в рамках динамического подхода к тестированию заслуживает работа Шайм-Сандера и Майерса [Shyam-Sunder\&Myers, 1999], которые продемонстрировали недостаточную мощность теста динамической теории компромисса не только на уровне интуиции, но и с помощью математической процедуры симуляции данных. В этой работе были использования данные базы Industrial Compustat за период с 1971-го по 1989 год, в результате исключения компаний, информация по которым была неполной, получилась небольшая выборка из 157 компаний.

И теория компромисса (уравнение (3)), и теория порядка финансирования (уравнение (4)) Шаймом-Сандером и Майерсом не отвергаются: коэффициент b в них значим и близок к 1. Получив такие результаты, исследователи пытаются понять, произошло ли это потому, что две теории каким-то образом сосуществуют параллельно, или же потому, что тесты не позволяют достаточно хорошо различить их.

Для проверки второй гипотезы авторы смоделировали два набора финансовых историй 157 компаний, покрывающих все 19 лет. В первом наборе финансовый рычаг определялся согласно теории порядка финансирования, исходя из значений внутреннего дефицита, с коррекцией на случайную ошибку. Во втором данные генерировались с помощью уравнения вида (3) для значений $(1-b)$ от 0,1 до 1 (с интервалом 0,1$)$. Целевое значение долга определялось как функция ряда факторов в рамках статичных моделей.

Для каждого из двух наборов данных строились модели (3) и (4). Результаты тестирования построенных моделей на значимость приведены в таблице 1.

Таблица 1.

Теории порядка финансирования и компромисса: симуляция и последующая проверка

\begin{tabular}{|c|c|c|c|}
\hline & \multicolumn{2}{|c|}{ Модель, по которой генерируются данныее } \\
\hline & & Теория компромисса & $\begin{array}{l}\text { Теория порядка } \\
\text { финансирования }\end{array}$ \\
\hline \multirow[t]{2}{*}{ Тестируемая модель } & $\begin{array}{l}\text { Теория } \\
\text { компромисса }\end{array}$ & $\begin{array}{l}\text { Тестируемая модель } \\
\text { работает }\end{array}$ & $\begin{array}{l}\text { Тестируемая модель } \\
\text { работает, хотя } \\
\text { авторегрессионное } \\
\text { поведение L не } \\
\text { подразумевалось }\end{array}$ \\
\hline & $\begin{array}{l}\text { Теория порядка } \\
\text { финансирования }\end{array}$ & $\begin{array}{lr}\text { Tестируемая } & \text { модель } \\
\text { не работает ни при } \\
\text { каком b } \\
\text { симуляции; } \\
\text { незначим }\end{array}$ & $\begin{array}{l}\text { Тестируемая модель } \\
\text { работает }\end{array}$ \\
\hline
\end{tabular}

При тестировании теории компромисса исследователи легко совершают «ошибку первого рода» - принимают теорию, хотя на самом деле она не имеет ничего общего с поведением фирм. Тестирование модели порядка финансирования от такой проблемы 
избавлено, поэтому использование уже такой простой модели, как (4), дает основания отдавать предпочтения этой теории

Лин и другие [Lin et.al., 2007] рассматривают интересную модификацию теории порядка финансирования, дополняя ее элементами поведенческих финансов - так называемой теорией избыточного оптимизма менеджеров. По мнению Хитона [Heaton, 2002], менеджеры склонны излишне позитивно оценивать результаты инвестиционных проектов и придерживаются мнения, что рынок оценивает эти проекты слишком низко. В результате проекты финансируются за счет внутренних средств, при их исчерпании фирма переходит к долгу, поскольку для ценообразования облигаций расхождение в оценках менеджеров и рынка менее существенно, и далее - к акциям, что согласуется с теорией порядка финансирования.

Лин и другие вводят в модель (4) дополнительную фиктивную переменную, отражающую уровень оптимизма менеджеров. Менеджер признается оптимистом, если в своих прогнозах он систематически завышает денежные потоки и прибыль компании. Результаты тестирования показывают, что у оптимистичных менеджеров в большей степени растет величина чистого долга при нехватке внутренних источников финансирования, то есть эти менеджеры менее склонны прибегать к выпуску акций.

Лооф [Loof, 2004], Банерджи и другие [Banerjee et.al., 2001], Ниворожкин [Nivorozhkin, 2004] используют для оценки динамической модели компромисса систему из трех регрессионных уравнений, в которой одновременно оцениваются оптимальный уровень финансового рычага и издержки приспособления к нему:

$$
\begin{aligned}
& L_{i t}=a+\left(1-b_{i t}\right) L_{i t-1}+b_{i t} L_{i t}^{*}+\varepsilon_{i t} ; \\
& L^{*}{ }_{i t}=a_{i t}+X_{i t} * \beta+r_{i t} ; \\
& b_{i t}=c_{i t}+Z_{i t} * d+u_{i t},
\end{aligned}
$$

где

$\mathrm{X}_{\mathrm{it}}$ - вектор факторов, определяющих оптимальный уровень финансового рычага согласно теории trade-off,

$\mathrm{Z}_{\mathrm{it}}$ - вектор факторов, определяющих скорость приспособления финасового рычага.

Банерджи проводит исследование по данным Великобритании и США, Лооф дополняет его проверкой модели по шведским данным и предполагает, что результаты должны значительно различаться, потому что финансовая система Швеции в большей степени ориентирована на банки, а не на привлечение средств с финансового рынка, как в Великобритании и США. Ниворожкин использует данные развивающихся экономик Восточной Европы - Чехии и Болгарии.

Похожую, но несколько более сложную модель, в которой дивидендная политика определяется одновременно с выбором структуры капитала, строит Адедеджи [Adedeji, 2001] по данным о британских компаниях.

Лооф, Банерджи, Ниворожкин и другие получают смешанные результаты, не позволяющие сделать выводы в пользу теории компромисса или порядка финансирования. Налоговые эффекты долга, как правило, не имеют объясняющей силы для уровня оптимального финансового рычага, размер компании и перспективы роста оказывают для одних стран положительное влияние на уровень финансового рычага, для других отрицательное. Ниворожкин отмечает интересную особенность развивающихся экономик Восточной Европы: доля материальных активов в общих оказывает отрицательное влияние на уровень долга. Автор объясняет это тем, что степень материальности активов важна для получения залогового финансирования, а оно в Чехии и Болгарии неразвито, поскольку стоимость залога крайней сложно взыскать в случае банкротства должника.

По мнению данной группы исследователей, скорость приспособления финансового рычага (b) определяется дистанцией между текущим и оптимальным уровнем рычага, темпом роста компании чем он больше, тем гибче компания может подстраивать финансовый рычаг), размер (чем компания крупнее, тем сложнее ей подстраиваться. На 
практике эти предположения не находят подтверждения: более крупные компании быстрее изменяют финансовый рычаг при необходимости, а темп роста не оказывает значимого влияния на скорость приспособления [Loof, 2004]. Как показывает Ниворожкин, скорость приспособления в развивающихся экономиках выше, чем в развитых. Это может объясняться тем, что компании на развивающихся рынках постоянно действуют в условиях неоптимальной структуры капитала и потому стараются как можно быстрее подстроиться под оптимальную, при этом сам целевой уровень финансового рычага меняется из-за экономической нестабильности.

Матеус и Терра [Mateus, Terra, 2005] тестируют динамические теории структуры капитала для большой выборки компаний: семь стран Латинской Америки плюс семь стран Восточной Европы, включая Россию. Авторы строят модель вида (5), (6), (7) в разностях, чтобы провести анализ влияния различных факторов на уровень наблюдаемого и оптимального финансового рычага. Они отмечают, что скорость приспособления к оптимальному значению долга у восточноевропейких компаний ниже, нежели у латиноамериканских. Основной проблемой их работы является незначимость большинства коэффициентов, которая быть следствием мультиколлинеарности, которая особенно сильна в разностях.

\section{4. Недостатки традиционных статических и динамических моделей. Новые подходы к тестированию теорий структуры капитала}

\section{1. Проблемы применения традиционных методов к тестированию статических и динамических моделей}

Как показывает проведенный в двух предыдущих разделах обзор работ, посвященный тестированию статических и динамических моделей структуры капитала, их результаты неоднозначны. В ряде исследований различия в уровнях финансового рычага между компаниями не могут быть объяснены ни теорией компромисса, ни теорией порядка финансирования. В других работах действие факторов на структуру капитала может быть интерпретировано в пользу как одной, так и другой теории, что заставляет задуматься о необходимости выработки некоего интегрированного подхода.

Мы считаем нужным отметить ряд недостатков используемых в такого рода исследованиях моделей, которые могли привести к неудачам в тестировании моделей.

Во-первых, эмпирические исследования теорий структуры капитала опираются на известное утверждение из теории корпоративных финансов о независимости инвестиционных и финансовых решений. Предполагается, что величина прибыли от основной деятельности (ЕВIT), которую способна генерировать фирма, не меняется при изменении уровня долговой нагрузки. На практике, как отмечает ряд авторов [Barton et.al., 1989], инвестиционные и финансовые решения взаимосвязаны.

Во-вторых, для расчета любой меры финансового рычага с использованием балансовых данных нужно привести отчетность к единообразному виду. Она также должна быть консолидирована, иначе финансовый рычаг будет занижен: компании могут осуществлять заимствования через дочерние компании, что не найдет отражения в неконсолидированном балансе. Авторы исследований, за исключением Раджана и Зингалеса [Rajan, Zingales, 1995], корректировок отчетности не проводят.

В-третьих, результаты регрессий могут страдать от мультиколлинеарности (например, прибыльность и перспективы роста компании, как правило, положительно коррелированы). Как результат, факторы, которые по результатам регрессии получились незначимыми, могут на самом деле быть значимы. И наоборот, могут наблюдаться кажущиеся (spurious) зависимости между данными, не отражающие практики принятия финансовых решений в компаниях (например, зависимость между рыночным финансовым рычагом и Q Тобина). 
В-четвертых, выборка компаний для тестирования может быть смещенной. Чтобы решить проблему с доступностью данных, исследователи выбирают наиболее известные и стабильные компании. Это вызывает смещение выборки в сторону более крупных компаний и компаний с более низким значением финансового рычага. Действует так называемое «смещение в сторону уцелевших» (survivor bias): компании, набравшие слишком много долгов, обанкротились, в результате чего большинство исследователей не включает их в выборку из-за недостатка данных.

В-пятых, несмотря на распространенность статического подхода к тестированию теорий компромисса и порядка финансирования, к его результатам нужно относиться с большой осторожностью. На практике существуют транзакционные издержки, препятствующие приспособлению фактического значения долга к оптимальному, причем для компаний развивающихся рынков они могут быть весьма значительными. Поэтому к результатам Бута и других [Booth et.al, 2001] и Чена [Chen, 2004], которые отвергают теорию компромисса в пользу теории порядка финансирования, нужно относиться с большой осторожностью.

Наконец, все тесты, направленные на проверку релевантности динамической теории компромисса, страдают недостатком мощности. Как показали Шайм-Сандер и Майерс [Shyam-Sunder\&Myers, 1999], исследователи, полагающиеся на их результаты, с большой вероятностью совершают ошибку второго рода и признают значимость динамической теории компромисса, в то время как авторегрессионное поведение финансового рычага может объясняться авторегрессионной динамикой фундаментальных переменных (финансового дефицита).

В связи с перечисленными недостатками традиционных исследований мы полагаем, что к их результатам нужно относиться с большой осторожностью. Перспективными направлениями проверки теорий компромисса и порядка финансирования, решающими часть проблем, являются динамические модели теории компромисса с горизонтом бездействий и метод изучения событий (event studies). Эмпирические исследования, выполненные в рамках этих подходов, представлены в следующих разделах обзора.

\section{2. Динамические модели теории компромисса с горизонтом бездействия}

В основе динамической модели с горизонтом бездействия, тестирование которой проводится в работах Фишера и других [Fischer et.al., 1989] и Стребулаева [Strebulaev, 2003], лежит теоретическая концепция, разработанная Кейном, Маркусом и Макдональдом [Каnе, Marcus, McDonald, 1984, 1985].

Базовая предпосылка работы Фишера и других состоит в том, что оптимальный уровень финансового рычага определяется в рамках компромиссной теории, то есть точкой равенства предельных выгод налогового щита и издержек банкротства. В силу существования издержек приспособления уровня долговой нагрузки к своему оптимальному значению фирмы будут осуществлять его не мгновенно, а постепенно, причем при небольших отклонениях фактического финансового рычага от оптимального, фирмы не будут производить подстройку, поскольку транзакционные издержки перевешивают выгоды от нее. Оптимальность подобной данной стратегии при выборе уровня финансового рычага фирмы была ранее продемонстрирована в теоретических работах Константинидеса [Constantinides, 1979] и Таксара и других [Taksar, 1983].

Фишер и другие предполагают, что рыночная стоимость собственного капитала является стохастическим процессом, в результате чего отношение балансовой стоимости долга к рыночной стоимости акций, то есть финансовый рычаг, также представляет собой стохастическую величину. Целью фирмы является максимизация стоимости в условиях присутствия корпоративных налогов и транзакционных издержек, в результате чего, как показывают авторы, подстройка уровня долга происходит только при выходе финансового рычага за определенные границы. 
Поведение фирмы при достижении границы определяется характеристиками транзакционных издержек. Авторы отвергают возможный вариант о том, что издержки пропорциональны изменению капитала, поскольку это привело бы к минимальным корректировкам структуры капитала для возвращения на границу, что не соответствует действительности. По мнению Фишера и других, более предпочтительным считается предположение о том, что по достижении границы весь старый долг погашается, а издержки пропорциональны новому. В итоге фирма от любой границы возвращается к одной и той же структуре капитала - изменения точечные во времени и скачкообразные.

При проведении симуляции данных на основе построенной модели Фишер и другие показывают, что при уровне транзакционных издержек в $1 \%$ стоимости фирмы и реалистичном значении других параметров наблюдается значительный диапазон бездействия. Так, при оптимальном соотношении долг - собственный капитал на уровне $62 \%$ границы диапазона бездействия составляют 29\% (нижняя) и 175\% (верхняя). Как следствие, статическая теория компромисса даст абсолютно неадекватное описание процедуры выбора уровня долга, поскольку она предполагает равенство наблюдаемого уровня долга оптимальному.

Для эмпирической проверки своей теории Фишер и другие использовали квартальные данные с III квартала 1977 года по IV квартал 1985-го из базы Industrial Quarterly COMPUSTAT (компании США). Горизонт бездействия оценивался ими как разница между максимальным и минимальным значением показателя за рассматриваемый период. В качестве оценки издержек банкротства авторы использовали бинарную фиктивную переменную в зависимости от отрасли компании [Titman, Wessels, 1983].

На основе построенной модели авторы вводят гипотезу о том, что более широкий диапазон бездействия характерен для малых фирм с низкой эффективной ставкой налога на прибыль, большой волатильностью рыночной стоимости активов и низкими издержками банкротства. Высокий уровень неопределенности стимулирует такие фирмы откладывать принятие решений об изменении структуры капитала, а слабое влияние налоговых эффектов и издержек банкротства на стоимость делает оптимальный финансовый рычаг нечувствительным к изменению условий внешней среды. Фишер и другие находят подтверждение этой гипотезе, используя в оценках обобщенный метод наименьших квадратов. Простой метод наименьших квадратов отвергается из-за присущей данным гетероскедастичности.

Впоследствии в работе Уэлча [Welch, 2004] было показано, что в реальности компании не приспосабливают уровень финансового рычага при изменении доходности собственного капитала, а значит, и его стоимости. В свете этого исследования подход Фишера и других вызывает определенные сомнения. Кроме того, Фишер и другие исходили из стандартной предпосылки о независимости инвестиционных и финансовых решений, которые, как показали Бартон и другие [Barton et.al., 1989], взаимосвязаны через недолговой налоговый щит.

В работе Стребулаева [Strebulaev, 2003] представлено перспективное направление компьютерной симуляции в анализе динамического поведения фирм, предложенное ранее Берком и другими [Berk et.al., 1999]. В работе показывается, что применяемые на сегодняшний день методы эмпирической проверки теорий структуры капитала дают зачастую неправильные выводы о связях между уровнем долговой нагрузки и характеристиками фирмы. Стребулаев показывает, что существующие тесты не дают возможности ответить на вопрос, говорят ли наблюдаемые отклонения финансового рычага от оптимального уровня о том, что показатель находится не на своем оптимальном уровне, или о том, что целевой уровень рассчитан неверно.

Теоретическая модель, использованная для компьютерной симуляции Стребулаевым, во многом опирается на разработанную Гольдштейном и другими [Goldstein et al., 2001] концепцию. Модель базируется на идее, что у компании есть заинтересованные лица, которые выставляют к ней финансовые требования: акционеры, кредиторы, государство. 
Величина данных требований моделируется в качестве стохастической величины. Рассматривается полная система налогообложения: налоги на прибыль, дивиденды и доходы. Как и в модели Фишера и других, компании производят активные действия по изменению структуры капитала на границах диапазона бездействия, которые Стребулаев называет точками рефинансирования. Агентские конфликты из рассмотрения исключаются: считается, что менеджеры действуют в интересах акционеров, максимизируя стоимость компании.

После построения динамической модели в рамках компромиссной теории Стребулаев производит моделирование данных на ее основе. Калибровка параметров производится на основе данных по рынку США, а именно на основе баз данных COMPUSTAT и CRSP. Далее на полученных данных автор строит регрессионные зависимости на основе метода наименьших квадратов, наиболее часто применяемые в эмпирических исследованиях по анализу. Цель этих действий состоит в том, чтобы определить, удастся ли стандартным эмпирическим тестам верно определить заложенные в модели предпосылки.

В результате автор находит четкую отрицательную связь между прибыльностью и финансовым рычагом. Аналогичное соотношение было обнаружено во многих других работах [Booth et.al., 2001; Chen, 2004; Norvaišienè, Stankevičienè, 2006; Declcoure, 2007], что было однозначно интерпретировано авторами в пользу верности теории порядка финансирования. В работе Майерса [Myers, 1993] данный аргумент назван наиболее убедительной эмпирической особенностью структуры финансирования и характеристик фирм, что свидетельствует об однозначном поражении компромиссной теории. Однако в работе Стребулаева данные были симулированы на основе модели, в которой изначально закладывалась положительная связь.

Автор приходит к выводу, что такое противоречие связано с тем, что стандартные методы оценки не учитывают динамическое поведение фирм и недооценивают значение точек рефинансирования. В моменты, когда компания находится в рамках горизонта бездействия, рост прибыльности повышает оценку будущих прибылей, а значит, приводит к росту рыночной стоимости компании, то есть снижает финансовый рычаг. В моменты изменений финансового рычага в точках рефинансирования связь между прибыльностью и рычагом находится в соответствии с компромиссной теорией. Однако стандартные тесты по пространственным выборкам фиксируют в большей степени первый эффект и, в результате, получают ложные выводы. Стребулаев получает выводы в пользу динамической компромиссной теории, в частности, подтверждается вывод Оплера и Титмана [Opler, Titman, 1994], Фамы и Френча [Fama, French, 2002] и ряда других исследователей о том, что финансовый рычаг демонстрирует тенденцию возвращения к среднему.

\section{3. Метод изучения событий (event study) в тестировании теории порядка финансирования}

Метод изучения событий сводится к анализу изменений стоимости компании, вызванных решениями о выпуске акций, облигаций или гибридных ценных бумаг. Этот метод позволяет проверить, действуют ли в реальности аргументы теории иерархии, возможности подтверждения или опровержения теории компромисса весьма ограничены. Это объясняется тем, что в основе теории иерархии лежат предположения о реакции рынка на выпуск тех или иных ценных бумаг, и именно эта реакция анализируется при использовании метода событий. В то же время тестирование теории компромисса требует анализа влияния определенных факторов на выбор структуры капитала, а не анализа реакции рынка на сообщения о выпуске ценных бумаг. Тем не менее результаты некоторых исследований позволяют сделать выводы относительно как теории иерархии, так и теории компромисса. 
Рассматриваемые работы основаны на анализе влияния выпуска гибридных ценных бумаг (конвертируемых облигаций и в отдельных случаях exchangeable bonds ${ }^{72}$ ) на цену акций. Конвертируемые облигации представляют собой облигации, которые по желанию их владельца могут быть обменены на заданное количество акций эмитента по истечении определенного срока с момента их выпуска. Кроме того, некоторые конвертируемые облигации могут быть принудительно обменены на акции по желанию эмитента - такие бумаги называются отзывными. Таким образом, конвертируемые облигации сочетают в себе свойства акций и облигаций. Поэтому выпуск конвертируемых облигаций представляет собой нечто среднее между привлечением акционерного и заемного капитала. В зависимости от характеристик этих ценных бумаг решение об их выпуске может по-разному восприниматься инвесторами. Очевидно, что эффект зависит от того, к какому инструменту (акциям или облигациям) ближе данный выпуск конвертируемых облигаций. Решение о выпуске конвертируемых облигаций является сигналом об изменении структуры капитала компании и может оказывать влияние на ее стоимость.

\subsection{1. Развитые рынки}

Бурлаку [Burlacu, 2000] рассматривает эффекты выпуска конвертируемый облигаций на цену акций (и соответственно на капитализацию компании). Автор указывает на то, что в предыдущих исследованиях по рынку США [Smith, 1986; Kuhlman and Radcliffe, 1992; Brennan and Her, 1993; Davidson, 1995 ${ }^{73}$ б была выявлена положительная зависимость между падением цены акций после объявления о выпуске конвертируемых облигаций и акционерным компонентом этих бумаг. Под акционерным компонентом понимается степень близости конвертируемой облигации к акции. Иными словами, чем ближе конвертируемая облигация по характеристикам к акции, тем сильнее снизится цена при объявлении о выпуске гибридных бумаг.

Для оценки акционерного компонента Бурлаку использует чувствительность стоимости конвертируемой облигации к изменениям цены акций той же компании (обозначается $\Delta$ ). Используется предпосылка о том, что конвертируемая облигация может быть представлены как сумма обычной облигации и варранта (ценной бумаги, дающей право на покупку определенного количества акций по определенной цене), а следовательно, чувствительность конвертируемой облигации совпадает с чувствительностью варранта. В оценке чувствительности варранта присутствует текущая цена акций, конверсионная цена (номинал облигации, деленный на цену конверсии), безрисковая ставка, стандартное отклонение доходности акций, количество лет до погашения, дивидендная доходность, накопленная вероятность при стандартном нормальном распределении. Значение величины $\Delta$ характеризует вероятность того, что облигация будет конвертирована в акции. Чем ближе значение $\Delta$ к единице, тем более чувствительна стоимость облигации к изменению цен акций и, следовательно, тем выше вероятность конвертирования и больше акционерный компонент. Приближение показателя $\Delta$ к нулю означает, что акционерный компонент невелик и конвертируемая облигация близка к обычной облигации.

Автор использует две даты анонсирования: получение разрешения на выпуск от комиссии по ценным бумагам (далее - $\mathrm{COB}$ ); появление официального сообщения о предстоящем выпуске в специализированном печатном издании (далее - BALO), разделяет компании по типу выпускаемых конвертируемых облигаций (долговые, смешанные, акционерные) позволяет выделить некоторые особенности. Так, долговые облигации выпускаются фирмами с относительно низким потенциалом роста. Акционерные облигации выпускают компании с высоким потенциалом роста. Это согласуется с теорией о

\footnotetext{
72 Здесь и далее под словом «облигация» понимаются именно гибридные ценные бумаги, если не указано иное.

${ }^{73}$ Burlacu R. New evidence on the pecking order hypothesis: the case of French convertible bonds//Journal of Multinational Financial Management. Vol. 10, 2000. P. 440.
}

Выпуск \#1(5), 2008 С Электронный журнал Корпоративные Финансы, 2008 
недоинвестировании, изложенной в статье Майерса [Myers, 1977] $]^{74}$, согласно которой, компании с высоким потенциалом роста должны предпочитать выпуск акций выпуску долговых инструментов. Автор также показывает, что компании, выпускающие долговые облигации, значительно крупнее, чем компании, выпускающие акционерные облигации. Это, в свою очередь, согласуется с гипотезой о том, что для крупных компаний характерны высокие агентские издержки свободного денежного потока ${ }^{75}$, а долг выполняет дисциплинирующую функцию.

Что касается изменений abnormal performance цен акций после выпуска, то оно снижается как после выпуска долговых облигаций, так и после выпуска акционерных. В большей степени снижение проявляется для акционерных облигаций - abnormal performance снижается с 3,37 до -5,87\%. Этот факт соответствует теории, изложенной в работе /Майерса и Майлуфа// [Myers \& Majluf, 1986], согласно которой менеджеры стремятся выпускать акции, когда они переоценены.

При проведении анализа с помощью метода событий автор оценивает изменения в abnormal returns в районе даты объявления о выпуске конвертируемых облигаций. При этом рассматривается как эффект для всей выборки, так и отдельно для компаний, выпускающих долговые, смешанные и акционерные облигации. Abnormal returns рассчитывается как разница между фактической доходностью акций и нормальной доходностью, оцененной с помощью рыночной модели. Данный подход является стандартным и используется во многих работах, поэтому подробно не рассматривается. Помимо abnormal returns (AR) за несколько дней рядом с датой объявления автор также рассчитывает средние abnormal returns (AAR) за определенные периоды рядом с датой объявления.

Для обеих дат (COB и BALO) реакция рынка по всей выборке является отрицательной. Для даты COB, реакция более сильная и значима на 1\% уровне значимости; для даты BALO, эффект слабее и значим только на 10\%-ном уровне значимости. Это может означать, что основная информация, как и предполагал автор, передается в момент СОВ. При разделении выборки по типам выпускаемых облигаций реакция на дату СОВ остается отрицательной и значимой для всех типов облигаций, а реакция на дату BALO зависит от типа - для акционерных облигаций реакция отрицательная, для смешанных - положительная, а для долговых - незначимая.

Для количественной оценки зависимости реакции рынка от величины акционерного компонента, автор строит регрессионную модель и показывает, что зависимость нелинейна. Вид зависимости говорит о том, что для смешанных облигаций в среднем наблюдается положительный эффект, а для акционерных и долговых - отрицательный. Полученные результаты, с точки зрения автора, могут объясняться дополненной моделью Майерса и Майлуфа Myers-Majluf, которая была предложена в статье Куни и Кэйли [Cooney and Kalay, $1993]^{76}$ и в которой говорится о возможности инвестирования в проекты с отрицательным NPV. Сам же автор дает следующее объяснение: для смешанных облигаций наблюдаются наиболее высокие премии (превышение цены, по которой продается облигация, над ее теоретической стоимостью); кроме того, поскольку компании, выпускающие смешанные облигации, обладают высоким потенциалом роста и высокой степенью асимметрии информации о ценности будущих проектов, выпуск акционерных или долговых облигаций мог бы повлечь высокие издержки неблагоприятного отбора и агентские издержки долга соответственно. В то же время выпуск смешанных облигаций позволяет обойти эти проблемы.

\footnotetext{
${ }_{75}^{74}$ Myers S.C. Determinants of Corporate Borrowing//Journal of Financial Economics. Vol. 5, 1977. Pp. $147-176$.

75 Jensen M. and Meckling W. Theory of The Firm: Managerial Behaviour, Agency Costs and Capital Structure//Journal of Financial Economics. Vol. 3, 1976. Pp. 305-360.

${ }^{76}$ Cooney J.W., Kalay A.Positive information from equity issue announcements// Journal of Financial Economics. Vol. 33, 1993. Pp. 149-172.
}

Выпуск \#1(5), 2008 
Для проверки выдвинутых предположений автор строит регрессионную зависимость average abnormal returns (AAR) от акционерного компонента и других факторов, оценивает ее с помощью МНК. Результаты подтверждают предположения.

Отдельно выделяется положительное влияние величины премии на реакцию рынка. По мнению автора, чем выше премия, тем выше вероятность того, что облигации в будущем будут конвертированы и премия будет перераспределена в пользу акционеров.

Основные выводы:

- $\quad$ В среднем чем больше акционерный компонент конвертируемой облигации, тем более негативная реакция рынка следует за объявлением о выпуске, что согласуется с теорией иерархии.

- $\quad$ Положительная реакция рынка на объявление о выпуске смешанных облигаций обусловлена уникальными характеристиками соответствующих компаний и может быть объяснена с помощью дополненной теории иерархии.

- $\quad$ Реакция рынка является результатом взаимодействия акционерных и долговых составляющих конвертируемых облигаций с одной стороны и премии - с другой.

При исследовании реакции рынка не только на объявление, но и на выпуск конвертируемых и обмениваемых ${ }^{77}$ облигаций Амманн, Фер и Заиц [Ammann, Fehr, Seiz, 2006] помимо анализа характера влияния на динамику цен акций объявления о выпуске и самого выпуска этих бумаг оценили зависимость реакции рынка от предшествующего поведения всего рынка в целом. ${ }^{78}$

Согласно результатам, рынок отрицательно реагирует на анонсирование выпуска - в день объявления Abnormal returns снижаются на 1,41\%. При этом за один и два дня до анонсирования наблюдаются положительные Abnormal returns. Авторы предполагают, что менеджеры делают объявление о выпуске облигации в тот момент, когда рост цен акций опережает рынок (рост рыночного индекса). Авторы также демонстрируют, что в период [0;1] наблюдается отрицательные cumulative Abnormal returns в размере -1,61\%, что говорит о том, что негативная реакция рынка частично имеет место после объявления о выпуске.

Авторы показали, что реакция рынка на выпуск облигаций отличается для немецких и швейцарских компаний. В обеих странах реакция отрицательная, однако в Германии снижение Abnormal returns более значительное. Также авторы показали, что реакция рынка зависит от типа выпускаемых облигаций: объявление о выпуске обмениваемых exchangeable облигаций влечет за собой более значительное снижение курса акций по сравнению с выпуском конвертируемых акций.

При тестировании зависимости реакции рынка от предшествующего поведения всего рынка в целом автор использовали разные временные периоды. Однако результаты практически не менялись, и авторы подтвердили результаты предыдущих исследований ${ }^{79}$, что реакция инвесторов меняется с течением времени и отрицательно зависит от акционерного компонента; рыночная же доходность, предшествовавшая объявлению о выпуске, наоборот, оказывает положительное влияние на реакцию рынка. Авторы объясняют это тем, что положительная динамика рыночных индексов говорит о хороших перспективах на будущее, что, в свою очередь, снижает неопределенность относительно будущего положения компании. В модель также были включены переменные величины компании, принадлежности к той или иной стране, а также типа выпускаемых облигаций.

Разделение выборки на выпуски конвертируемых и exchangeable облигаций изменяет значения коэффициентов: для конвертируемых облигаций реакция рынка отрицательно

\footnotetext{
${ }^{77}$ Обмениваемые облигации могут быть обменены на акции других компаний. Как правило, компания-эмитент владеет некоторым пакетом акций той компании, на которую может быть обменена exchangeable облигация.

${ }^{78}$ В выборке присутствуют немецкие и швейцарские компании. Дата анонсирования выпуска определяется как дата появления информации в информационной системе Reuters. Дата выпуска соответствует дате объявления условий выпуска. Акционерный компонент $\Delta$ и его интерпретация остается такой же, как у Буркалу.

${ }_{79}$ Choe et al. [1993], Bayless and Chaplinsky [1996], Lewis et al. [2003].
}

Выпуск \#1(5), 2008

(C) Электронный журнал Корпоративные Финансы, 2008 
зависит от акционерного компонента, в то время как для exchangeable облигаций реакция рынка не зависит от доли акционерного компонента. Это объясняется тем, что даже при высоких значениях $\Delta$ для exchangeable облигаций их обмен на акции не вызовет роста акционерного капитала у эмитента, поскольку обмен производится на акции другой компании.

\section{Основные выводы:}

- $\quad$ Рынок отрицательно реагирует на объявление о выпуске как конвертируемых, так и exchangeable облигаций при этом реакция в момент выпуска отсутствует.

- $\quad$ Реакция рынка отрицательно зависит от доли акционерного компонента, что согласуется с теорией иерархии.

- $\quad$ Реакция рынка зависит от того, в какой стране происходит выпуск облигаций.

• $\quad$ Реакция рынка зависит от предшествующей динамики рынка в целом.

\subsection{2. Развивающиеся рынки}

В силу специфики рынков развивающихся экономик компромиссная концепция, теория порядка финансирования и агентские модели порой не могут объяснить выбор структуры капитала. Неразвитость финансовой системы часто не позволяет компаниям пользоваться такими инструментами, как долгосрочные банковские кредиты, для финансирования инвестиций. Поэтому компании начинают следовать новой теории порядка финансирования: нераспределенная прибыль - выпуск акций - заемное финансирование, когда нераспределенная прибыль является самым быстрым доступным и надежным источником. $^{80}$

Кастильо [Castillo, 2004] изучал реакцию курса чилийских ценных бумаг на объявления о размещении акций или облигаций компании на фондовом рынке на примере 172 эмиссий. Автор использовал следующую модель:

(8) $\varepsilon_{i t}=R_{i t}-E\left[R_{i t} \mid X_{t}\right]$,

где

$\varepsilon_{i t}$ - реакция курса на объявление об эмиссии в период $\mathrm{t}$ (abnormal returns),

$R_{i t}$ - нормальный курс ценной бумаги в период $\mathrm{t}$,

$E\left[R_{i t} \mid X_{t}\right]$ - условное мат. ожидание курса ценной бумаги в период $\mathrm{t}$ на информационном множестве $\mathrm{X}$.

Вследствие того что на развивающихся рынках бумаги торгуются не каждый день, Кастильо использовал разные подходы для оценки нормального курса ценной бумаги в период $\mathrm{t}$, однако результаты получились идентичные:

При эмиссии долговых ценных бумаг курс не меняется статистически значимо после объявления об эмиссии; при эмиссии долевых ценных бумаг происходит статистически значимое отрицательное изменение курса.

Аластаир Марсден [Alastair Marsden, 2000] исследовал реакцию курса ценных бумаг на объявление о выпуске акций среди акционеров (rights issues) в Новой Зеландии в период с 1976-го по 1994 год. Выборка содержит 88 rights issues. Также учитывая неежедневность торговли акциями на фондовом рынке, автор понял, что чем больше относительный размер эмиссии, тем сильнее реакция курса на объявление, реакция курса на объявление отрицательная.

Применение методологии event study как для развитых, так и для развивающихся рынков позволяет сделать вывод о том, что реакция рынка на выпуск ценных бумаг

\footnotetext{
${ }^{80}$ Delcoure N., 2006. The determinants of capital structure in transitional economies // International Review of Economics and Finance 16, 2007. Pp. 400-415. 
согласуется с теорией pecking order. При этом реакция курса на объявление об эмиссии долговых ценных бумаг выявляется не для всех развивающихся стран, что, как правило, объясняется неразвитостью рынка долгового финансирования в развивающихся странах.

\section{Заключение}

Несмотря на существование обширного круга работ, посвященных тестированию теорий структуры капитала, ни теория компромисса, ни теория порядка финансирования до сих пор не нашли полного подтверждения на практике.

Ряд исследователей [Booth et.al., 2001; Chen, 2004] приходят к выводу, что для развивающихся экономик релевантна теория порядка финансирования, или «новая теория порядка финансирования», в которой при исчерпании нераспределенной прибыли для инвестиций осуществляет выпуск акций, поскольку долговой рынок неразвит. Хотя Кобхэм и Сабраманиам [Cobham, Subramaniam, 1998] утверждают, что такое положение дел естественно ввиду слабости системы бухучета и аудита в развивающихся странах, усиливающей асимметрию информации, другие исследователи [Strebulaev, 2003] демонстрируют, что теория порядка финансирования может приниматься ввиду игнорирования динамической природы теории компромисса, которая на самом деле описывает поведение фирм.

В то же время сами тесты динамической теории компромисса страдают недостатком мощности. Как продемонстрировали Шайм-Сандер и Майерс [Shyam-Sunder, Myers, 1999], авторегрессионное поведение финансового рычага может объясняться вовсе не теорией компромисса, но авторегрессионной динамикой фундаментальных переменных, в частности финансового дефицита.

Новые подходы к тестированию теорий структуры капитала - калибровка динамических моделей с горизонтом бездействия и метод изучения событий, — к сожалению, малоприменимы для российского рынка. Для получения надежных оценок параметров динамических моделей требуется большой набор данных и их внутренняя стабильность. Число российских компаний, раскрывающих всю необходимую информацию, невелико, акции многих компаний остаются неликвидными. Меняющиеся регулятивные требования и общее макроэкономическое окружения не позволяют надеяться на получения стабильных оценок параметров для калибровки моделей.

Применение методики изучения событий требует большого количества наблюдений (выпуск облигаций либо дополнительный выпуск акций). IPO российских компаний представляют меньший интерес в рамках этого метода, поскольку акции выпускаются впервые; кроме того, многие российские компании проводят первичные размещения на зарубежных площадках. Акции российских компаний часто неликвидны, что не позволяет отследить изменения курсов при объявлении решения о выпуске ценных бумаг.

Перечисленные выше ограничения позволяют нам предположить, что применение новых подходов к тестированию теорий структуры капитала российских компаний в ближайшие несколько лет будет невозможно. На наш взгляд, особый интерес представляет тестирование теории порядка финансирования в динамическом аспекте (изменение финансового рычага в ответ на динамику внутреннего дефицита компании) по методике, описанной Шайм-Сандером и Майерсом [Shyam-Sunder, Myers, 1999]. К тестированию теории компромисса как в статическом, так и в динамическом аспектах нужно относиться с большой осторожностью; в первом случае - ввиду наличия транзакционных издержек приспособления к оптимальной структуре капитала, во втором - ввиду недостаточной мощности используемых тестов. 


\section{Список литературы}

1. Дамодаран А. Инвестиционная оценка: Инструменты и методы оценки любых активов. М.: Альпина Бизнес Букс, 2005.

2. Adedeji A. A cross-sectional test of pecking order hypothesis against static trade-off theory on UK, University of Birmingham Working Paper, 2001.

3. Almeida H., Philippon T. The Risk-Adjusted Cost of Financial Distress, 2006. www.afajof.org/afa/forthcoming/3421.pdf

4. Ammann M., Fehr M., Seiz R. New evidence on the announcement effect of convertible and exchangeable bonds//Journal of Multinational Financial Management. Vol. 16, 2006, pp. 4363.

5. Andrade G., Kaplan S.N. How Costly is Financial (Not Economic) Distress? Evidence from Highly Leveraged Transactions that Became Distressed// NBER Working Paper 6145, 1997.

6. Auerbach A.S. Real determinants of corporate leverage. Published i n: Friedman, B.M. Corporate capital structures in the United States// University of Chicago Press, Chicago.

7. Banerjee S., Heshmati A., Wihlborg C. The dynamics of capital structure// Stockholm School of Economics Working Paper Series in Economics and Finance, № 333.

8. Barton H., Sundaram A. An empirical test of stakeholder theory predictions of capital structure// Financial Management, Vol. 18, 1989, pp. 36-44.

9. Baxter N.D., Nevins D., Cragg J.G. Corporate Choice Among Long-Term Financing Instruments // The Review of Economics and Statistics, Vol. 52, № 3 (Aug.), 1970, pp. 225-235.

10. Bayless, M., Chaplinsky, S. Is there a window of opportunity for seasoned equity issuance?//Journal of Finance,. Vol. 51, 1996, pp. 253-278.

Berk J., Green R., Naik V. Optimal Investment, Growth Options, and Security Returns // Journal of Finance, Vol. 54, 1999, pp. 1553-1607.

11. Borio C. Leverage and financing of non-financial companies: an international perspective // Economic Papers 27, Bank for International Settlements.

12. Bradley M., Jarrell G.A., Kim E.H. On the Existence of an Optimal Capital Structure: Theory and Evidence// The Journal of Finance, Jul., 1984, pp. 857-878.

14. Burlacu R. New evidence on the pecking order hypothesis: the case of French convertible bonds//Journal of Multinational Financial Management, Vol. 10, 2000, pp. 439-459.

15. Castillo A. The announcement effect of bond and equity issues: evidence from Chile//Estudios de Economía, Vol. 31, 2004, pp. 177-205.

16. Chen J.J. Determinants of Capital Structure of Chinese-listed Companies// Economic Change and Restructuring, Vol. 38 (1), 2004, pp.1-35.

17. Choe, H., Masulis, R.W., Nanda,V. Common stock offerings across the business cycle: theory and evidence// Journal of Empirical Finance, Vol. 1, 1993, pp. 3-31.

18. Chun S. Issues in Emerging Markets: Determinants, Effects, and Stock Market Performance of IPOs in Korea// Working paper, 2002.

19. Cobham D.,Subramaniam R. Corporate finance in developing countries: New evidence for India// World Development, Vol. 26, 1998, pp. 1033-1047.

20. Constantinides G. Multiperiod Consumption and Investment Behavior with Convex Transactions Costs// Management Science, Vol. 25, 1979, pp. 1127-1237.

21. Cooney, J.W., Kalay, A.Positive information from equity issue announcements// Journal of Financial Economics, Vol. 33, 1993, pp. 149-172.

22. Delcoure N. The determinants of capital structure in transitional economies// International Review of Economics and Finance, Vol. 16, 2007, pp. 400-415.

23. Donaldson G. Corporate debt capacity: a study of corporate debt policy and the determination of corporate debt capacity// Boston: Harvard Graduate School of Business Administration, 1961.

24. Fama E.F., French K.R. Testing Trade-Off and Pecking Order Predictions about Dividends and Debt// The Review of Financial Studies, Vol. 15(1), 2002, pp. 1-33. 
25. Fischer E., Heinkel R., Zechner J. Optimal Dynamic Capital Structure Choice: Theory and Tests// Journal of Finance, Vol. 44, 1989, pp. 19-40. Paper

26. Frank M.Z., Goyal V.K. Capital Structure Decisions// EFA 2004 Maastricht Meetings

№ 6. 2464; Tuck Contemporary Corporate Finance Issues III Conference Paper, 2002.

27. Frankel A., Montgomery J. Financial structure: an international perspective// Brookings Papers on Economic Activity, Vol. 1,1991, pp. 257-297.

28. Gilson S.C. Transaction Coasts and Capital Structure Choice: Evidence from Financially Distressed Firms// The Journal of Finance, Vol.52 (1), 1997, pp.161-196.

29. Goldstein R., Ju N., Leland H. An EBIT-Based Model of Dynamic Capital Structure// The Journal of Business, Vol. 74, 2001, pp. 483-512.

30. Graham J., Harvey C. The theory and practice of corporate finance: evidence from the field// Journal of Financial Economics, Vol. 60, 2001, pp. 187-243.

31. Grinblatt M., Titman S. Financial markets and corporate strategy// The McGraw-Hill Companies, 2002.

32. Heaton J.B. Managerial Optimism and Corporate Finance// Financial Management, Vol. 31, 2002, pp. 33-45.

33. Hovakimian A., Opler T., Titman S. The debt-equity choice// Journal of Financial and Quantitative Analysis, Vol. 36(1), 2001, pp. 1-24.

34. Jalilvand A., Harris R.S. Corporate behavior in adjusting to capital structure and dividend targets: an econometric study// Journal of Finance, Vol. 39, 1984, pp. 127-145.

35. Jensen M. and Meckling W. Theory of The Firm: Managerial Behaviour, Agency Costs and Capital Structure//Journal of Financial Economics, Vol. 3, 1976, pp. 305-360.

36. Kaiser K. Corporate restructuring and financial distress: an international view of bankruptcy laws and implications for corporations facing financial distress// INSEAD Working paper, 1994.

37. Kane A., Marcus A., McDonald R. Debt policy and the rate of return to leverage// Journal of Financial and Quantitative Analysis, Vol. 20, 1985, pp. 47-499.

38. Kane A., Marcus A., McDonald R. How Big is the Tax Advantage to Debt?// Journal of Finance, Vol. 39, 1984, pp. 841-853.

39. Lewis, C.M., Rogalski, R.J., Seward, J.K. Industry conditions, growth opportunities and market reactions to convertible debt financing decisions//Journal of Banking \& Finance, Vol. 27, 2003, pp. 153-181.

40. Lin Y., Hu S., Chen M. Testing pecking order prediction from the viewpoint of managerial optimism: Some empirical evidence from Taiwan// Pacific-Basin Finance Journal, Vol. 13, 2007, pp. 523-546.

41. Long, M.S. and Malitz, J. The investment financing nexus: some empirical evidence// Midland Corporate Finance Journal, Vol.3, 1985, pp. 53-59.

42. Loof H. Dynamic Optimal Capital Structure and Technical Change// Structural Change and Economic Dynamics, Vol. 15, 2004, pp.449-468.

43. Marsden A. Shareholder wealth effects of rights issues: Evidence from the New Zealand capital market./Pacific-Basin Finance Journal, Vol. 8, 2000, pp. 419-442.

44. Marsden N., Veeraraghavan Z. The Relationship between Debt and Nontradable Shares in China, http://www.efmaefm.org/0EFMAMEETINGS/EFMA\%20ANNUAL\%20MEETINGS/2007Vienna/Papers/0403.pdf

45. Marsh P. Choice Between Equity and Debt: An Empirical Study// The Journal of Finance, Vol. 37(1), 1982, pp. 121-144.

46. Mateus C., Terra P. Capital Structure and Debt Maturity: Evidence from Emerging Markets// Working Paper. http://www.fma.org/SLC/Papers

47. Miller M. Debt and taxes// Journal of Finance, Vol. 32, 1977, pp. 261-275. 
48. Modigliani F., Miller M. The cost of capital, corporation finance and the theory of investment// American Economic Review, Vol. 48, 1958, pp. 261-297.

49. Myers S., Majluf N. Corporate financing and investment decisions when firms have information that investors do not have// Journal of financial economics, Vol. 13, 1984, pp. 187-221.

50. Myers S.. Still searching for optimal capital structure// Journal of Applied Corporate Finance, Vol. 6, 1993, pp. 4-14.

51. Myers S.C. Determinants of Corporate Borrowing//Journal of Financial Economics, Vol. 5, 1977, pp. 147-176.

52. Nivorozhkin E. The Dynamics of Capital Structure in Transition Economies// Economics of Planning, Vol. 37, 2004, pp. 25-45.

53. Norvaišienè R., Stankevičienè J. The Interaction of Internal Determinants and Decisions on Capital Structure at the Baltic Listed Companies// Engineering Economics, Vol. 52(2), 2007, pp. $7-17$.

54. Opler T., Titman S. The debt-equity choice: an analysis of issuing firms// Working paper, Boston college, Boston.

55. Rajan G.R., Zingales L. What Do We Know about Capital Structure? Some Evidence from International Data// The Journal of Finance, Vol.50(5), 1995, pp. 1421-1460.

56. Ross $\mathrm{S}$. The determinants of financial structure: the incentive signaling approach// Bell Journal of Economics, Vol. 8, 1977, pp. 23-40.

57. Shyam-Sander L., Myers S.C. Testing Static Tradeoff against Pecking Order Models of Capital Structure// Journal of Financial Economics, Vol. 51, 1999, pp. 219-244.

58. Strebulaev, I. Do Tests of Capital Structure Theory Mean What They Say?// Working paper. London Business School, 2003.

59. Taggart R.A. A model of corporate financing decisions// Journal of Finance, Vol. 32, 1977, pp. 1467-1484.

60. Taksar K., Assaf D. Diffusion Model for Optimal Portfolio Selection in Presence of Brokerage Fees// Technical Report No. 4, Stanford University Department of Operations Research, 1983.

61. Taub A.J. Determinants of the Firm's Capital Structure// The Review of Economics and Statistics, Vol. 57(4), 1975, pp. 410-416.

62. Titman S., Wessels R. The Determinants of Capital Structure Choice// Journal of Finance, Vol. 43, 1988, pp. 1-19.

63. Tong G., Green C.J. Pecking Order or Trade-off Hypothesis? Evidence on the Capital Structure of Chinese Companies// Applied Economics, Vol. 37, 2004, pp. 2179-2189.

64. Weiss L. A. Bankruptcy resolution: Direct costs and violation of priority of claims// Journal of Financial Economics, Vol. 27, 1990, pp. 255-311.

65. Welch I. Capital Structure and Stock Returns// Journal of Political Economy, Vol. 112, 2004, pp. 106-131.

66. White M.J. Corporate bankruptcy: a US-European comparison// Working paper, University of Michigan 1993.

67. Xiaoqiu W. China capital market: non-tradable shares and liquidity reform// China People University Press, 2004.

68. Zou H., Xiao J. Z. The financing behaviour of listed Chinese firms// The British Accounting Review, 38, 2006, pp. 239-258. 